\title{
Rice yellow stunt virus activates polyamine biosynthesis to promote viral propagation in insect vectors by disrupting ornithine decarboxylase antienzyme function
}

\author{
Xiao-Feng Zhang ${ }^{\dagger}$, Tianbao Zeng ${ }^{\dagger}$, Yunjie Xie, Yuemin Zheng, Huanqin Wang, Hanbin Lin, \\ Zongwen Wang \& Taiyun Wei"
}

State Key Laboratory of Ecological Pest Control for Fujian and Taiwan Crops, Institute of Plant Virology, Fujian Agriculture and Forestry
University, Fuzhou 350002, China

Received August 7, 2020; accepted November 13, 2020; published online January 8, 2021

\begin{abstract}
Intracellular polyamines (putrescine, spermidine, and spermine) have emerged as important molecules for viral infection; however, how viruses activate polyamines biosynthesis to promote viral infection remains unclear. Ornithine decarboxylase 1 (ODC1) and its antienzyme 1 (OAZ1) are major regulators of polyamine biosynthesis in animal cells. Here, we report that rice yellow stunt virus (RYSV), a plant rhabdovirus, could activate putrescine biosynthesis in leafhoppers to promote viral propagation by inhibiting OAZ1 expression. We observed that the reduction of putrescine biosynthesis by treatment with difluormethylornithine (DFMO), a specific nontoxic inhibitor of ODC1, or with in vitro synthesized dsRNAs targeting ODC1 mRNA could inhibit viral infection. In contrast, the supplement of putrescine or the increase of putrescine biosynthesis by treatment with dsRNAs targeting OAZ1 mRNA could facilitate viral infection. We further determined that both RYSV matrix protein $\mathrm{M}$ and $\mathrm{ODC} 1$ directly bind to the ODC-binding domain at the C-terminus of OAZ1. Thus, viral propagation in leafhoppers would decrease the ability of OAZ1 to target and mediate the degradation of ODC1, which finally activates putrescine production to benefit viral propagation. This work reveals that polyamine-metabolizing enzymes are directly exploited by a vector-borne virus to increase polyamine production, thereby facilitating viral infection in insect vectors.
\end{abstract}

rice yellow stunt virus, leafhopper, polyamines, viral infection, plant virus

Citation: Zhang, X.F., Zeng, T., Xie, Y., Zheng, Y., Wang, H., Lin, H., Wang, Z., and Wei, T. (2021). Rice yellow stunt virus activates polyamine biosynthesis to promote viral propagation in insect vectors by disrupting ornithine decarboxylase antienzyme function. Sci China Life Sci 64, 1522-1532. https://doi. org $/ 10.1007 / \mathrm{s} 11427-020-1846-8$

\section{INTRODUCTION}

The polyamines putrescine (Put), spermidine (Spd), and spermine (Spm) are small, positively charged molecules present in both prokaryotic and eukaryotic cells. Polyamines are involved in myriad fundamental cellular processes, including cell proliferation, ion channel regulation, DNA conformation, transcription, protein synthesis, and protein-

$\dagger$ Contributed equally to this work

*Corresponding author (email: weitaiyun@fafu.edu.cn)
RNA interactions (Igarashi and Kashiwagi, 2010, 2019; Kusano et al., 2008; Michael, 2016; Tabor and Tabor, 1984). The steady-state levels of cell polyamines are elaborately regulated by the enzymes involved in their metabolism, providing a highly controlled feedback mechanism. These enzymes include ornithine decarboxylase (ODC1) and its antienzyme OAZ1, spermidine/spermine-N1-acetyl transferase (SSAT), and spermine oxidase (SMO) (Fong et al., 1976; Matsufuji et al., 1995; Pegg, 2008). ODC1, a critical enzyme in the biosynthesis of polyamines, is regulated by a negative feedback loop involving OAZ1, which inhibits 
ODC1 activity and accelerates its degradation in a ubiquitinindependent manner to limit production of endogenous polyamines (Beenukumar et al., 2015; Fong et al., 1976; Matsufuji et al., 1995; Palanimurugan et al., 2014). In animal cells, ornithine is decarboxylated via the action of ODC1 to produce Put, the precursor for synthesis of Spd and Spm (Michael, 2016). Importantly, difluormethylornithine (DFMO), a specific nontoxic inhibitor of ODC1, reduces cellular or organismal polyamine levels (Fozard et al., 1979; Metcalf et al., 1978). Polyamines are not only essential for cell growth and proliferation, but also required for the infection cycles of both DNA and RNA viruses, including human disease pathogens such as cytomegalovirus, Zika virus, MERS coronavirus, and rabies virus (Mounce et al., 2017).

Viruses rely on polyamines at numerous stages of viral infection cycle, including virion packaging, genome replication, gene transcription, and viral protein translation (Mounce et al., 2017). For example, double-stranded DNA (dsDNA) viruses recruit polyamines for maintaining genomic DNA conformation during the replication process (Gibson et al., 1984; Pohjanpelto et al., 1988; Williamson, 1976). For RNA viruses, polyamines seem to be more important for viral gene expression and genome replication (Mounce et al., 2016b; Tuomi et al., 1982). To take advantage of polyamines in host cells, some viruses have evolved mechanisms to modulate polyamine metabolic pathways. Paramecium bursaria chlorella virus 1, a DNA pathogen of green algae, encodes an ODC-like protein to satisfy its demand for a high concentration of polyamines during replication (Morehead et al., 2002). Some viruses also regulate the activities of host enzymes of the polyamine biosynthesis pathway. For example, the decreased polyamine content was noted in cells infected with herpes simplex virus type 1 (family Herpesviridae), possibly due to the suppressed ODC1 expression (Tyms et al., 1979). However, the molecular mechanism underlying the modulation of polyamine biosynthesis through activation of the enzymes to benefit viral replication in host cells remains elusive.

Many viral pathogens that cause global health and agricultural problems are transmitted via insect vectors including mosquitoes, thrips, aphids, planthoppers, and leafhoppers. For example, highly infectious animal viruses such as Zika virus and dengue virus, and plant viruses such as rice stripe virus, are transmitted by insect vectors in a persistent-propagative manner (Morrison, 2014; Fauci and Morens, 2016; Wei and Li, 2016). Persistent-propagative viruses, when ingested by their insect vectors, firstly replicate and assemble their progeny virions in the midgut epithelium, from where they disseminate to the midgut visceral muscles. The viruses move from the midgut muscles into the hemolymph and finally into the salivary glands, from where they can be introduced into plant hosts (Hogenhout et al., 2008; Wei and
Li, 2016). Even in very-well-studied mosquito systems, little is known about how viruses activate cellular polyamine biosynthesis to promote viral persistent infection in insect vectors.

Recent emerging plant rhabdoviruses transmitted by insect vectors in a persistent-propagative manner cause substantial agricultural losses (Fang et al., 2019; Di et al., 2014; Liu et al., 2018; Yang et al., 2017, 2018). Rice yellow stunt virus (RYSV), an insect-transmitted rice nucleorhabdovirus, was first identified in 1965 in Taiwan and Guangdong provinces of China (Chiu et al., 1965; Fan et al., 1965). It caused a serious reduction in rice yields in Asia (Hibino, 1996). In the field, this virus is transmitted by the rice green leafhopper Nephotettix cincticeps in a persistent-propagative manner (Chiu et al., 1965; Fan et al., 1965). RYSV has a bulletshaped morphology, ranging from 45 to $100 \mathrm{~nm}$ in width and 130 to $350 \mathrm{~nm}$ in length (Chiu et al., 1965; Fan et al., 1965). Its genome contains a negative-sense, single-strand RNA of 14,030 nucleotides, which encodes seven proteins in the

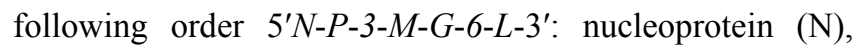
phosphoprotein $(\mathrm{P})$, putative movement protein (P3), matrix protein (M), glycoprotein (G), P6, and large RNA polymerase (L) (Fang et al., 1994; Huang et al., 2003, 2005; Luo et al., 1998; Luo and Fang, 1998; Wang et al., 2018). RYSV $\mathrm{M}$, a small (20-25 kD) self-association protein, associates with genomic nucleocapsids and condenses ribonucleoprotein (RNP) complex cores to form non-enveloped, bulletshaped particles (Luo and Fang, 1998; Wang et al., 2018, 2019, 2020). We recently observed that RYSV $M$ on the surface of non-enveloped virions could directly interact with insect axonal microtubules to mediate viral axonal transport in the central nervous system (CNS) of $N$. cincticeps (Wang et al., 2019). Furthermore, the interaction of RYSV $\mathrm{M}$ and the neural factor Hikaru genki homolog of $N$. cincticeps limits viral spread in vector CNS (Wang et al., 2020). Here, we further determine that RYSV M could directly interact with $\mathrm{OAZ1}$ of $N$. cincticeps and negatively regulate its expression, which causes the higher ODC1 accumulation and more Put production to benefit viral propagation in viruliferous insect vectors. For the first time, to our knowledge, we reveal how polyamine-metabolizing enzymes are directly exploited by a vector-borne virus to modulate polyamine production to facilitate viral propagation of its insect vectors.

\section{RESULTS}

\section{RYSV M interacts with OAZ1 but not with ODC1}

In our previous study, we showed that the interaction of RYSV M with actin played an important role in viral spread in the CNS of leafhopper $N$. cincticeps (Wang et al., 2019). To identify other insect factors that interact with RYSV M, 
we used $\mathrm{M}$ as bait to screen the yeast cDNA library from $N$. cincticep. The C-terminal region of leafhopper OAZ1 was identified from the yeast two-hybrid screening. We then cloned the full-length of OAZ1 and ODC1 genes based on the bioinformatics analysis of leafhopper RNA-seq result (Figures S1 and S2 in Supporting Information). OAZ1 of $N$. cincticeps was decoded from two open reading frames (ORFs) by ribosomal frame shifting, which is the classical feature of OAZ1 reported in other species (Figure S1 in Supporting Information). To facilitate the following studies of the interaction between OAZ1 and RYSV M, we created an OAZ1 frame shift mutant to encode the full length of OAZ1 protein. In the following assay, all OAZ-1 clones indicated the full length of OAZ1 protein.

We next tested whether OAZ1 and ODC1 of $N$. cincticeps were able to interact with RYSV-encoded proteins. For a yeast two-hybrid system, we made constructs expressing different RYSV-encoded proteins (N, P, P3, M, P6, and G) fusing with Gal4 DNA binding domain (BD) as the bait fusion proteins or with Gal4 activation domain (AD) as the prey proteins, respectively. Similarly, we generated the bait and prey constructs with OAZ1 or ODC1. Among the six RYSV-encoded proteins, only $M$ interacted with OAZ1 but not with ODC1 (Figure 1A). As expected, OAZ1 also interacted with ODC1 (Figure 1A). A glutathione S-transferase (GST) pull-down experiment further confirmed that RSYV M specifically interacted with OAZ1 (Figure 1B). Furthermore, we infected Sf9 cells with recombinant baculoviruses containing RYSV M, OAZ1, or ODC1 and examined the localization of these proteins by immunofluorescence microscopy. When expressed individually, $\mathrm{M}$ was associated with the plasma membrane, OAZ1 was distributed diffusedly in the cytoplasm, and ODC1 was localized in the nucleus (Figure 1C 1-III). Coinfection results showed that OAZ1 and $\mathrm{M}$ aggregated to form punctate inclusions in the cytoplasm (Figure 1C IV), while OAZ1 was recruited to the nucleus together with ODC1 (Figure 1C VI). However, M did not colocalize with ODC1 (Figure 1C V).

Since both RYSV M and ODC1 interacted with OAZ1, we next wanted to identify the exact domain of OAZ1 to which $\mathrm{M}$ or ODC1 bound. Based on the analysis of the characteristic ODC-binding domain (114-211 aa) at the Cterminus of OAZ1, we constructed a series of truncation mutants and carried out yeast two-hybrid analyses to investigate the binary interactions of OAZ1 mutants with the full-length M or ODC1 (Figure 1D). The results indicated that both RYSV M and ODC1 interacted with the ODCbinding domain of OAZ1 (Figure 1D). This implied that RYSV M competed with ODC1 for binding to the same domain of OAZ1. Together, RYSV infection may modulate polyamine biosynthesis by affecting the expression of polyamine-metabolizing enzymes in $N$. cincticeps through M-OAZ1 interaction.
RYSV infection increases production of Put in $N$. cincticeps

We then investigated whether RYSV infection could activate polyamine production of $N$. cincticeps. In general, insect polyamine Put is biosynthesized via the activity of ODC1, which is the first rate-limiting enzyme in Put biosynthesis and regulated by OAZ1 (Figure 2A). Put is subsequently converted to Spd and Spe via their respective synthases SRM and SMS (Figure 2A). Increasing evidence suggests that polyamines are involved in viral infection of host cells (Mounce et al., 2017). To determine the profile of polyamine accumulation during infection by RYSV of its insect vector $N$. cincticeps, we examined the concentrations of three polyamines, Put, Spd and Spm, by ultra-performance liquid chromatography tandem mass spectrometry (UPLC-3Q-MS) at different time points after RYSV infection. From 4 days post viral infection (dpi) of insect vectors, the Put concentration in RYSV-infected $N$. cincticeps, compared with the uninfected controls group, significantly increased (Figure 2B). However, RYSV infection did not significantly affect the accumulation levels of Spd or Spm (Figure 2B). This result combined with the above results suggests that RYSV infection activates the biosynthesis of Put in $N$. cincticeps.

\section{RYSV infection modulates the expression of polyamine- metabolizing enzymes OAZ1 and ODC1}

We next assessed how viral infection affected the expression of OAZ1 and ODC1 in the polyamine biosynthesis pathway over the time course of RYSV infection in its insect vectors. We first analyzed RYSV infection of insect vectors at different time points. RT-qPCR and Western blot assays showed that the expression of RYSV N and M could be clearly detected at $4 \mathrm{dpi}$ and then increased during viral infection of $N$. cincticeps at the transcript and protein levels (Figure 3A). More importantly, at 4 and 6 dpi, RT-qPCR and Western blot assays showed that OAZ1 expression was decreased, while ODC1 expression was increased at the transcript and protein levels during viral infection of leafhopper vectors (Figure 3B). Thus, it appeared that RYSV infection inhibited the OAZ1 expression but upregulated ODC1 expression, which may facilitate polyamine production in viruliferous insect vectors.

\section{Knocking down OAZ1 or ODC1 expression affects in- fection of RYSV in $N$. cincticeps}

Next we determined the functions of two key enzymes OAZ1 and $\mathrm{ODC} 1$ in the polyamine biosynthesis pathway during viral infection of $N$. cincticeps. We knocked down the expression of OAZ1 and ODC1 during RYSV infection, using RNA interference (RNAi) by microinjecting dsRNAs tar- 

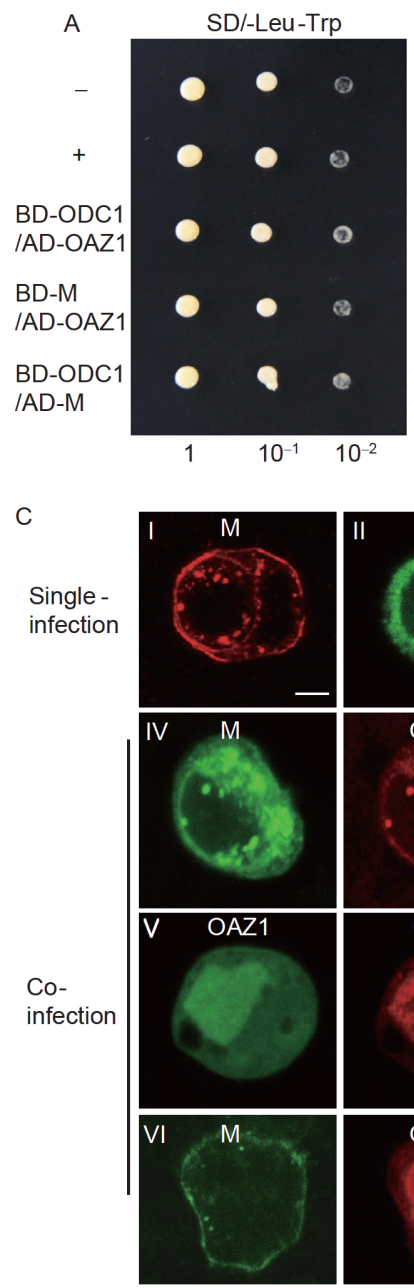
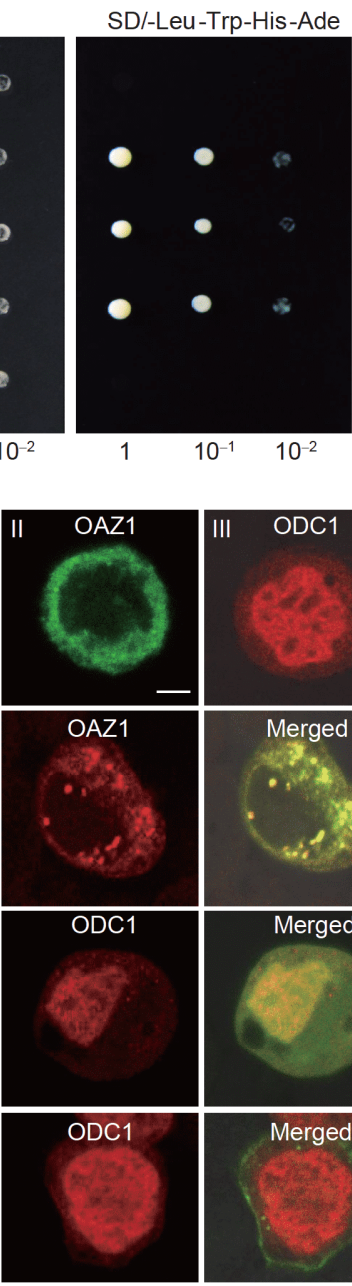
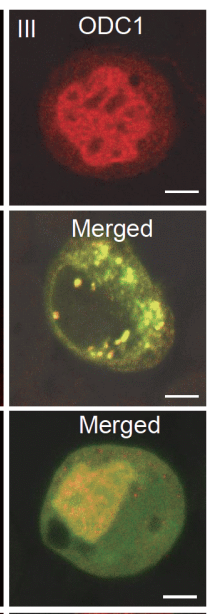

Merged

$\mathrm{B}$
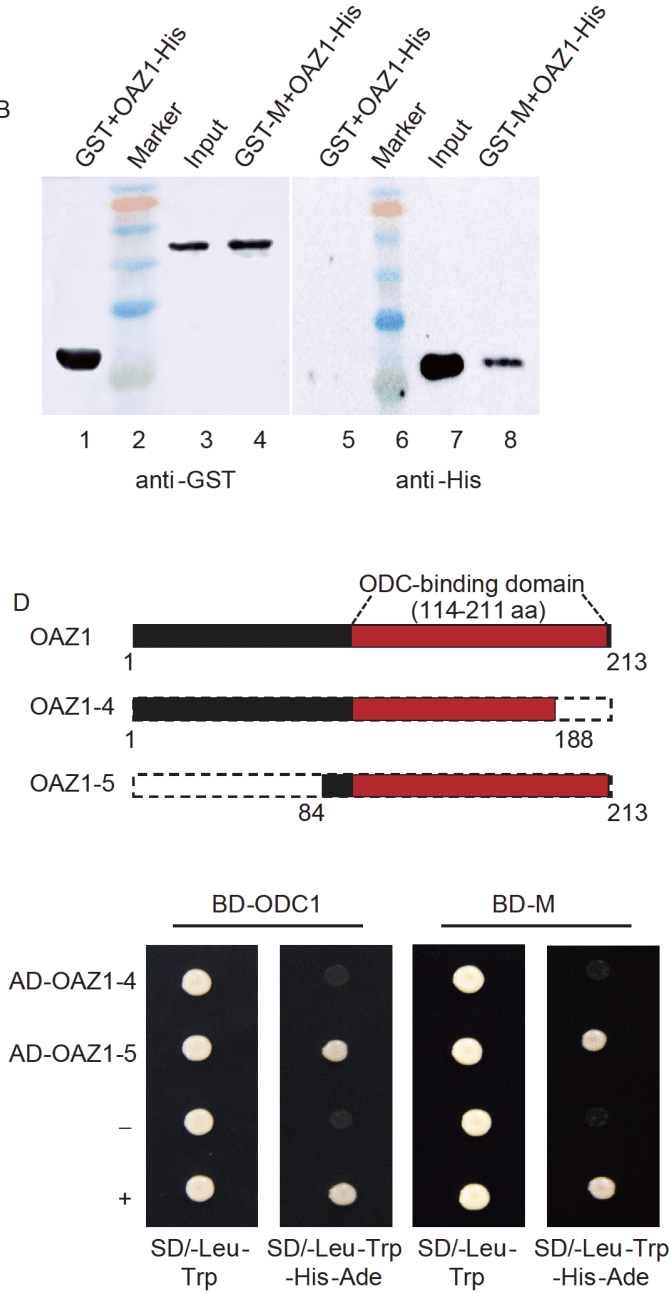

Figure 1 Interactions among RYSV M, OAZ1, and ODC1. A, Yeast two-hybrid assay of the interactions among RYSV M, OAZ1 and ODC1. The transformed cells were plated at different dilutions on non-selective (-Leu-Trp) and selective (-Leu-Trp-Ade-His). pGBKT7-53/pGADT7-T was used as the positive control $(+)$. pGBKT7/pGADT7-P was used as negative controls $(-)$. Serially diluted yeast cultures were shown. B, RYSV M was capable of interacting with OAZ1 in pull-down assay. Glutathione beads loaded with purified GST protein (lane 1, lane 5) or GST-fused M (GST-M) (lane 3, lane 7) were incubated with His-tagged recombinant OAZ1 (OAZ1-His). Following incubation, the amounts of GST-M and OAZ1-His in the reaction mixtures (input) and those bound to the beads (GST pull-down) were determined by immunoblotting using anti-GST and anti-His antibodies. C, Interactions among RYSV M, OAZ1 and ODC1 in Sf9 cells. I-III, At 30 hours post-infection (hpi), Sf9 cells infected with the recombinant bacmids containing M, OAZ1 or ODC1 were fixed and stained with M-rhodamine (red, I), OAZ1-FITC (green, II), or ODC1-rhodamine (red, III), respectively. IV, At 30 hpi, Sf9 cells coinfected with the recombinant bacmids containing M or OAZ1 were fixed and stained with M-FITC (green) and OAZ1-rhodamine (red). V, At 30 hpi, Sf9 cells co-infected with the recombinant bacmids containing OAZ1 or ODC1 were fixed and stained with OAZ1-FITC (green) and ODC1-rhodamine (red). VI, At 30 hpi, Sf9 cells co-infected with the recombinant bacmids containing M or ODC1 were fixed and stained with M-FITC (green) and ODC1-rhodamine (red). Scale bars, $15 \mu \mathrm{m}$. D, Yeast two-hybrid assay of the interaction domains of OAZ1 with RYSV M or ODC1. Diagram showing the different OAZ1 deletion mutant constructs used in two-hybrid assays to map the region involved in associating with M or ODC1. The predicted ODC1-binding domains (114-211 aa) of OAZ1 are shown in red.

geting OAZ1 (dsOAZ1) or ODC1 (dsODC1) mRNA. At 6 days after inoculation, the significant reduction in OAZ1 transcript level by dsOAZ1 treatment led to higher accumulation of ODC1 compared with the control group (dsGFP) (Figure 4A I, II, 4B II). Furthermore, we also detected the concentration of Put under treatment with dsOAZ1, and observed that the knockdown of OAZ1 expression remarkably promoted the production of Put (Figure 4A III). Thus, the dsOAZ1 treatment efficiently knocked down the in vivo expression of OAZ1 (Figure 4A I), thereby increasing the accumulation levels of ODC1 and intracellular Put content (Figure 4A III, 4B III). In contrast, knockdown of ODC1 expression by dsODC1 treatment did not significantly change the expression profile of OAZ1 (Figure 4A II), but led to the reduced accumulation of Put content (Figure 4A III). Thus, we determined that OAZ1 and ODC1 of $N$. cincticeps have the conserved roles in modulating polyamine biosynthesis. 
A

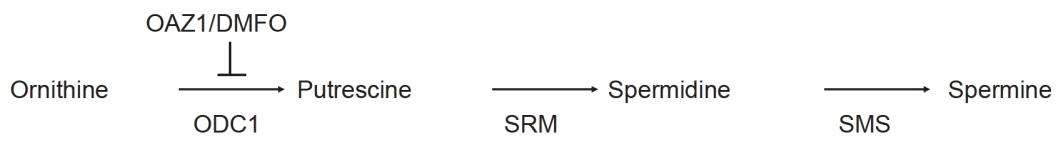
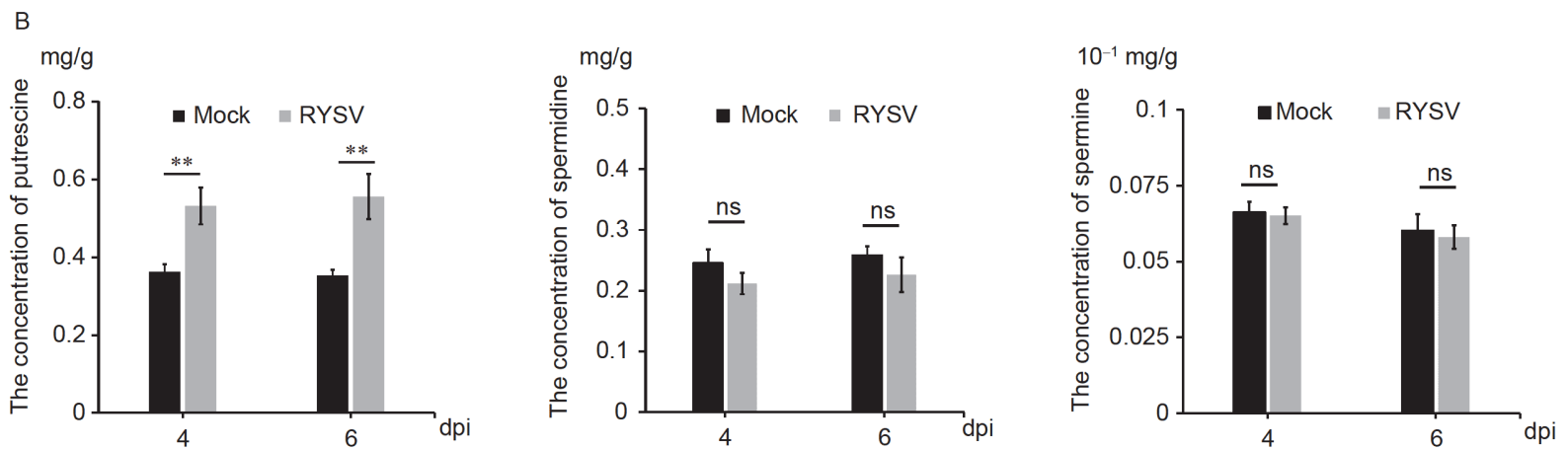

Figure 2 Concentrations of polyamines during infection of $N$. cincticeps by RYSV. A, Scheme illustrating the de novo synthesis pathway of polyamines and relevant enzymes and inhibitors used in this study. SRM, Spermidine synthase. SMS, Spermine synthase. B, N. cincticeps nymphs microinjected with RYSV were collected at 4 and 6 dpi, respectively. The intracellular polyamines were separately extracted and examined by UPLC-3Q-MS assay. $* * P \leqslant 0.01$ using a one-tailed Student's $t$ test. Error bars represent \pm SEM.

A 1

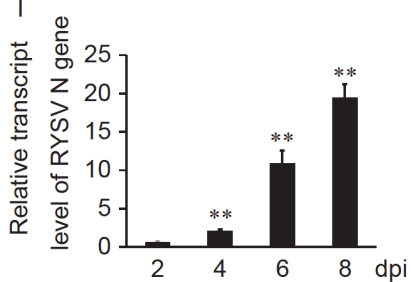

B I

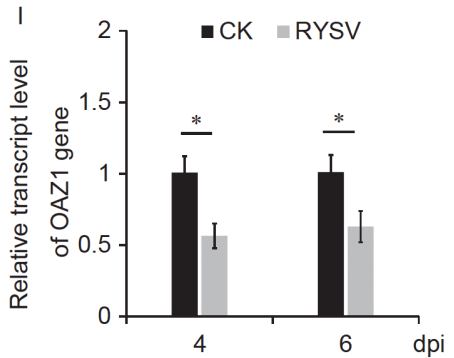

II
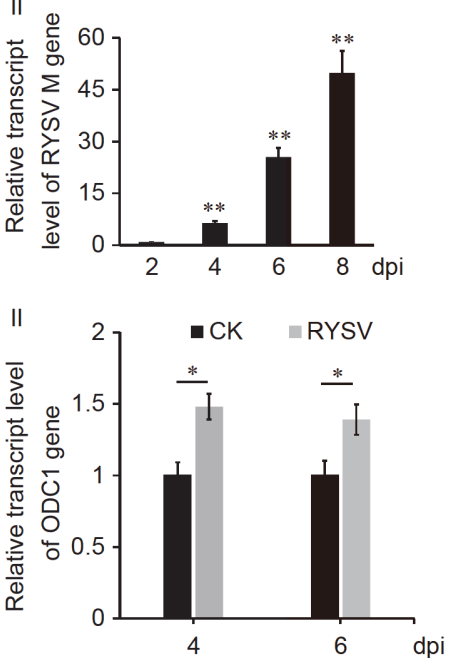

III

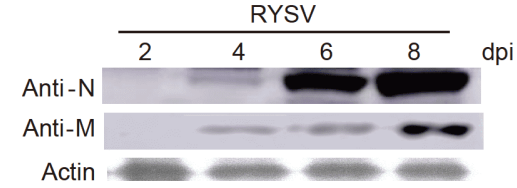

III

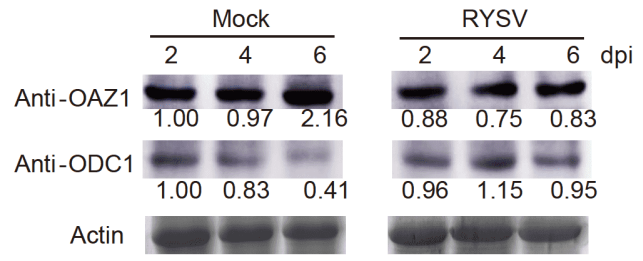

Figure 3 RYSV infection affected the expression of OAZ1 and ODC1 in N. cincticeps. A, Gene expression of RYSV during viral infection. I-II, Transcription levels of $\mathrm{N}$ or $\mathrm{M}$ genes were analyzed by RT-qPCR assay at 2, 4, 6, and 8 dpi. III, Accumulations of RYSV N or M proteins were detected by immunoblotting with specific antibodies against $\mathrm{N}$ or $\mathrm{M}$ at 2, 4, 6, and 8 dpi. Insect actin was used as an internal control. B, Expression of OAZ1 and ODC1 during RYSV infection. $N$. cincticeps nymphs infected with RYSV were collected at different time points of RYSV infection (4 and 6 dpi) for total RNA extraction and protein extraction. I-II, Transcript levels of OAZ1 or ODC1 genes were analyzed by RT-qPCR assay. III, Accumulations of OAZ1 or ODC1 proteins were detected by immunoblotting with specific antibodies against OAZ1 or ODC1. Insect actin was used as an internal control. Bands were quantified by densitometry scanning by using Quantity One software. ${ }^{*} P \leqslant 0.05, * * P \leqslant 0.01$ using a one-tailed Student's $t$ test. Error bars represent \pm SEM.

The effects of dsOAZ or dsODC1 treatment on infection by RYSV were assessed using RT-qPCR and Western blot assays. As expected, at 6 days after inoculation, knockdown of OAZ1 promoted the accumulation of RYSV N and M, while knockdown of ODC1 inhibited the accumulation of RYSV N and M in viruliferous $N$. cincticeps (Figure 4B). Recently, we observed that RYSV could systemically infect the CNS of its $N$. cincticeps vectors (Wang et al., 2019). At 6 days after inoculation, immunofluorescence microscopy showed that RYSV had spread extensively throughout the whole CNS in dsOAZ1-treated insects, while viral infection was restricted to a limited region of the CNS in dsODC1treated insects (Figure 4C). Together, these results confirmed that RYSV activated and elevated Put biosynthesis levels to promote viral propagation by inhibiting OAZ1 expression but increasing ODC1 expression in $N$. cincticeps.

\section{High intracellular Put concentration in $N$. cincticeps fa- cilitates infection by RYSV}

We next asked whether polyamine depletion in $N$. cincticeps caused by in vitro feeding of DFMO could inhibit the pro- 

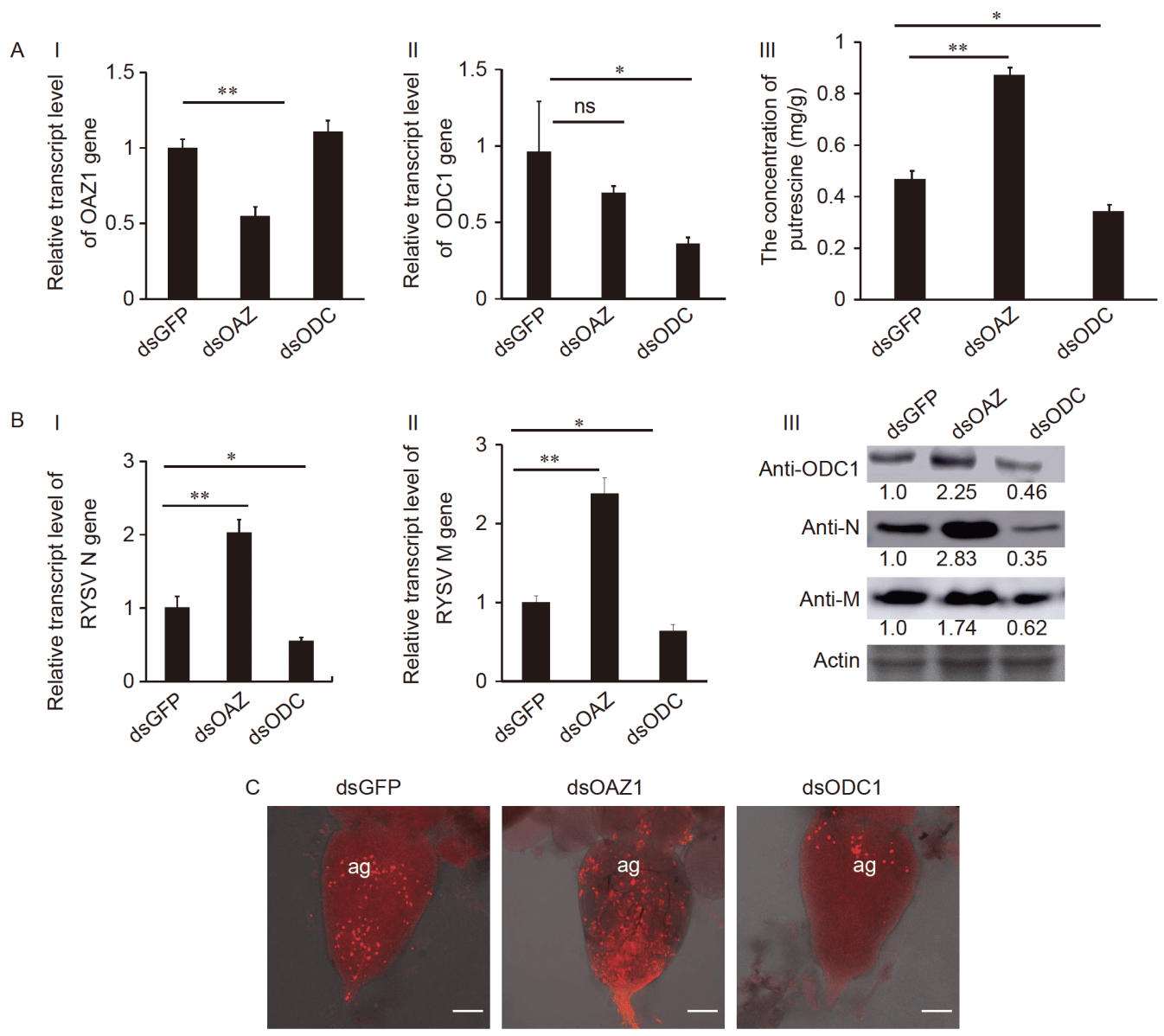

Figure 4 Modulation of polyamine biosynthesis by knockdown of ODC1 or OAZ1 expression affected the infection of $N$. cincticeps by RYSV. I-II, Transcription levels of OAZ1 or ODC1 genes of $N$. cincticeps after microinjection with different dsRNAs (dsGFP, dsOAZ1, or dsODC1), as revealed by RTqPCR assay. III, the concentrations of Put under different dsRNAs treatments were detected via UPLC-3Q-MS assay. B, N. cincticeps nymphs were microinjected with dsRNAs and RYSV solution. I-II, At 6 dpi, transcription levels of N and M genes of RYSV under different dsRNAs treatments were analyzed by RT-qPCR assay. III, Accumulations of ODC1, N and M proteins were detected by immunoblotting with specific antibodies against ODC1, N or M. Insect actin was used as an internal control. Bands were quantified by densitometry scanning using Quantity One software. C, RYSV infection of the internal organs of $N$. cincticeps under different dsRNAs treatments. At $6 \mathrm{dpi}$, the insect central nervous system was immunolabeled for M-rhodamine and examined by immunofluorescence microscopy. ag, abdominal ganglion. Scale bars, $100 \mu \mathrm{m}$. Bands were quantified by densitometry scanning by using Quantity One software. ${ }^{*} P \leqslant 0.05, * * P \leqslant 0.01$ using a one-tailed Student's $t$ test. Error bars represent \pm SEM.

pagation of RYSV. We first tested the effect of DFMO on the biosynthesis of polyamines in an in vitro feeding experiment. Two groups of healthy third-instar $N$. cincticeps nymphs were raised on food supplemented with or without DFMO $\left(10 \mathrm{mmol} \mathrm{L}^{-1}\right)$, respectively. UPLC-3Q-MS assay showed that the concentrations of polyamines Put, Spd and Spm were significantly reduced after the treatment of DFMO (Figure $5 \mathrm{~A})$.

To clarify the roles of polyamines during RYSV infection, we raised two groups of RYSV-infected third-instar $N$. cincticeps nymphs on food supplemented with or without DFMO $\left(10 \mathrm{mmol} \mathrm{L}^{-1}\right)$, respectively. At 4 days post in vitro feeding experiment, the accumulated levels of RYSV N, M, and $\mathrm{P}$ were assessed via RT-qPCR and immunoblotting assays. We observed a significant reduction in accumulation of these viral structural proteins in the DFMO-treated group compared with the untreated group (Figure 5B and C). We further determined whether the supplement of polyamines could facilitate viral infection. Three groups of RYSV-infected $N$. cincticeps nymphs were raised with food supplemented with Put, Spd, or Spm, respectively. As anticipated, we observed that only Put, but not Spd or Spm, was able to obviously elevate the accumulation levels of these viral structural proteins in insect vectors (Figure 5B and C). Thus, adequate Put contents in $N$. cincticeps are necessary for RYSV infection. Together, these results further demonstrated that RYSV activate the polyamine biosynthesis pathway to benefit its own propagation by $N$. cincticeps.

\section{DISCUSSION}

Host cell metabolism can be modulated by viral infection, affecting viral survival or clearance. Intracellular polyamine 

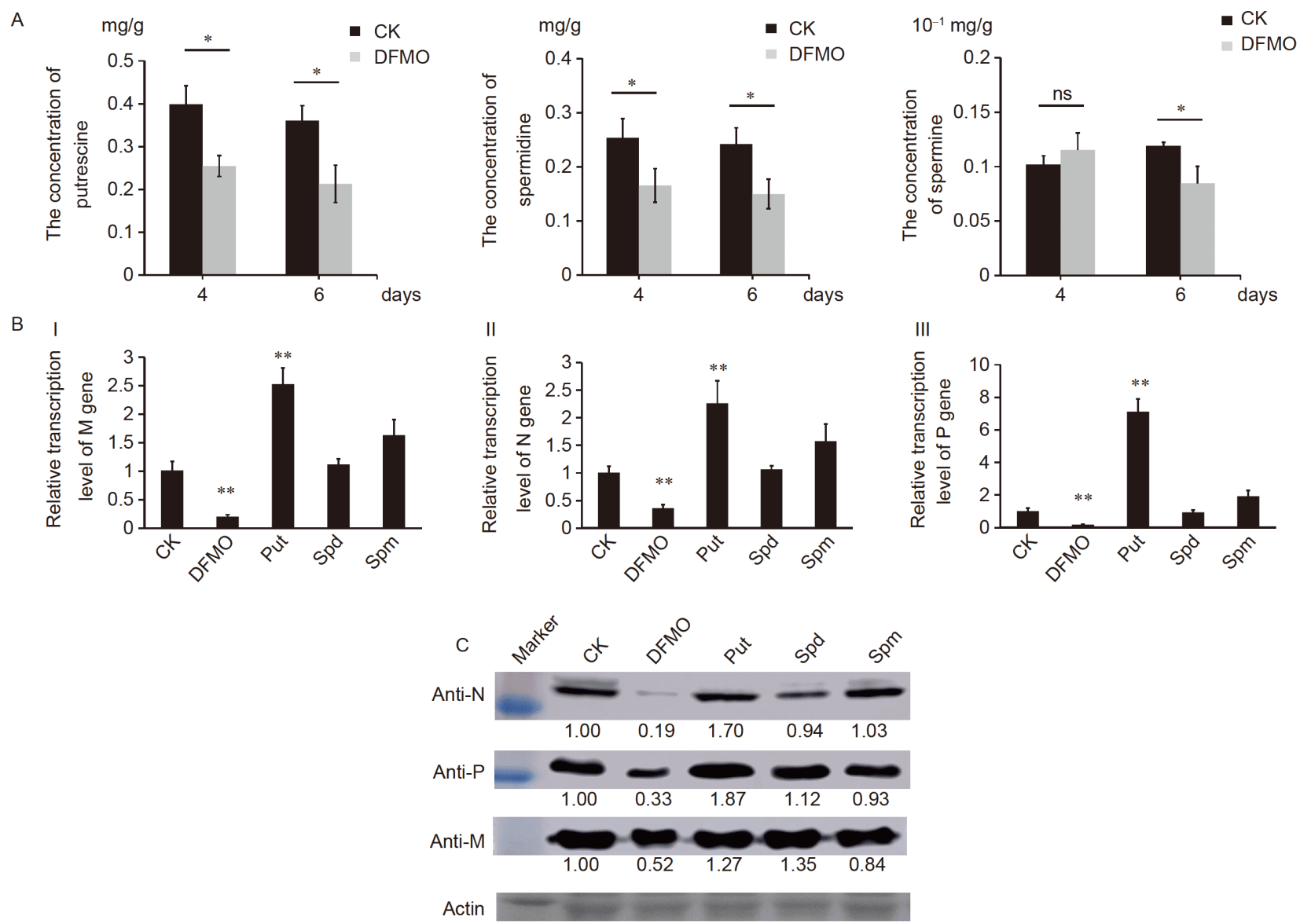

Figure 5 In vitro feeding of $N$. cincticeps with polyamines or DFMO affected the infection of $N$. cincticeps by RYSV. A, $N$. cincticeps nymphs were fed $10 \%$ sucrose solution with or without $10 \mathrm{mmol} \mathrm{L}^{-1}$ DFMO. Polyamines were extracted and measured after 4 and 6 days. B, N. cincticeps nymphs infected with RYSV solution were fed with $10 \%$ sucrose solution containing DFMO $\left(10 \mathrm{mmol} \mathrm{L}^{-1}\right)$ or one of three polyamines $\left(0.1 \mathrm{mmol} \mathrm{L}^{-1}\right)$. Relative transcript levels of the N (I), P (II), and M (III) genes in the different treated groups were analyzed using RT-qPCR assay. C, Accumulations of RYSV N, P, or M proteins in $N$. cincticeps under different treatments were detected by immunoblotting with specific antibodies against $\mathrm{N}$, P, or M. Insect actin was used as an internal control. Bands were quantified by densitometry scanning using Quantity One software. ${ }^{*} P \leqslant 0.05, * * P \leqslant 0.01$ using a one-tailed Student's $t$ test. Error bars represent \pm SEM.

metabolism is not only essential for the growth of normal cells, but also involved in different steps of viral infection process (Igarashi and Kashiwagi, 2010, 2019; Michael, 2016; Mounce et al., 2016a; Mounce et al., 2016b; Mounce et al., 2017). Many important insect-transmitted viral pathogens such as Zika virus, Chikungunya virus, and Dengue virus require polyamines for their propagation in mammal hosts (Mounce et al., 2016a; Mounce et al., 2016b; Olsen et al., 2016); how these viruses mediate the polyamines pathway for their infection in insect vectors remains unclear. In this study, we report that a rhabdovirus, RYSV, relies on the high concentration of Put for efficient vial propagation in its leafhopper vector, $N$. cincticeps. Viral infection significantly upregulates ODC1 expression but inhibits OAZ1 expression, which would facilitate the conversion of ornithine to Put. In vitro feeding of Put increases viral propagation, while the decreased Put accumulation after DFMO treatment inhibits viral propagation. Meanwhile, knockdown of OAZ1 expression increases viral propagation, while knockdown of
ODC1 expression inhibits viral propagation Taken together, we report that a vector-borne virus, RYSV, can increase Put production to benefit its own propagation in leafhopper vectors by reducing OAZ1 expression and elevating ODC1 expression (Figure 6).

It is noteworthy that previous work has focused on whether polyamines contribute to viral replication or translation (Mounce et al., 2017); however, how polyamine-metabolizing enzymes can be directly hijacked by viruses for regulation of polyamine homeostasis to facilitate viral infection remains poorly understood. Here, we further show that among the six RYSV-encoded proteins, only the M interacts with OAZ1, the ODC1 antizyme. Furthermore, both RYSV $\mathrm{M}$ and ODC1 interact with the same ODC-binding domain at the C-terminus of OAZ1. During the propagation of the nucleorhabdoviruses in the nuclei of insect vectors and plant hosts, the RNP cores are assembled within the viroplasm matrix in the nucleic, and then $M$ proteins recognize and condense RNP cores to assemble the bullet-shaped or ba- 


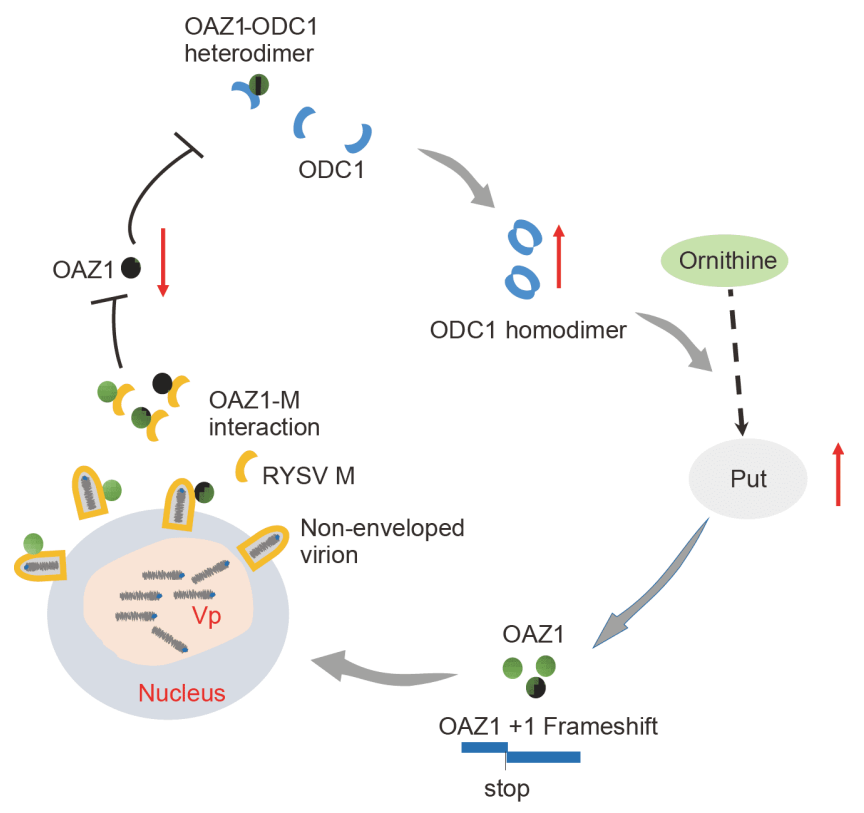

Figure 6 A model of RYSV M-modulated the increase of Put production to benefit viral propagation in leafhopper vectors by reducing OAZ1 expression and elevating ODC1 expression. Once RYSV particles successfully enter the leafhopper cell, M proteins are expressed at the early stage of RYSV infection. M proteins interact with OAZ1 proteins and promote its degradation. The reduction of OAZ1 proteins in RYSV-infected cells impair the degradation of ODC1 proteins and increase the accumulation of ODC1. More ODC1 proteins promote the biosynthesis of polyamines, which in turn facilitates infection by RYSV.

cilliform non-enveloped virions at the nuclear membrane (Wang et al., 2018; Jackson and Li, 2016; Jackson et al., 2018; Zhou et al., 2019). Our electron microscopic observation has shown that abundant non-enveloped bulletshaped virions of RYSV budded from the nuclear membrane into the cytoplasm in the CNS of $N$. cincticeps (Wang et al., 2019). We thus deduce that the highly efficient propagaion of RYSV in $N$. cincticeps would lead to the recruitment of abundant OAZ1 proteins to the assembled non-enveloped virons or expressed $M$ proteins at the nuclear membrane or in the cytoplasm of insect vectors, thereby decreasing the OAZ1 contents in virus-free regions for targetting ODC1 in the nucleus. Furthermore, RYSV propagation possibly also stimulates the activity of OAZ1 inhibitor (Palanimurugan et al., 2014), which will be investigated further. According to the homeostatic feedback regulation of polyamines, it is reasonable that the decreased expression of OAZ1 during RYSV infection in turn impairs the ability of OAZ1 to target ODC1 for ubiquitin-independent degradation, thereby resulting in higher ODC1 level, which further promotes the conversion of ornithine to Put. Together, our results depict a regulation network in which RYSV-encoded M protein hijacks OAZ1 to accelerate the biosynthesis of Put for viral propagation in insect vectors. Many rhabdoviruses, such as rabies virus (RABV) and vesicular stomatitis virus (VSV), have been extensively shown to require polyamines for the different stages of their propagation in host cells (Mounce et al., 2017). Furthermore, distantly related rhabdovirus M proteins have very similar structures for condensing the RNP cores, giving rigidity to the viral structures (Harty et al., 1999; Okumura and Harty, 2011; Finke and Conzelmann, 2003). Thus, it will be meaningful to investigate whether our discovery is a common mechanism shared by other rhabdoviruses.

Polyamines function in the transcription, translation, and cell cycling (Igarashi and Kashiwagi, 2010, 2019; Kusano et al., 2008; Michael, 2016; Tabor and Tabor, 1984). Viruses similarly rely on intracellular polyamines for transcription, translation, and nucleic acid packaging (Mounce et al., 2017; Firpo and Mounce, 2020). Thus, the requisition of cellular polyamine for virus infection may affect various cellular processes and insect growth. Rhabdoviruses such as RYSV, rice stripe mosaic virus, RABV and VSV are neurotropic and can infect the CNS of their insect vectors or hosts (Wang et al., 2019, 2020; Kakooza-Mwesige et al., 2019). Here, we determine that virus-activated Put production would be beneficial for RYSV infection of CNS of $N$. cincticeps, suggesting the high concentration of polyamines in CNS cells. Given the neurotropic characteristic of RYSV, whether high concentrations of Put caused by RYSV infection can affect the behaviors and growth of the insects is a topic worthy of further study. It is likely that this general mechanistic framework will be applicable to other vector-borne rhabdoviruses, possibly leading to the development of novel antiviral strategies that target this mechanism.

\section{MATERIALS AND METHODS}

\section{Insects, viruses, and antibodies}

A population of the non-viruliferous leafhopper $N$. cincticeps was collected from Yunnan province in southern China and reared on rice seedlings in a cage under the following conditions: $28^{\circ} \mathrm{C}$ with $75 \% \pm 5 \%$ humidity and $16 / 8 \mathrm{~h}$ light/dark cycle. RYSV-infected rice samples were propagated via transmission by $N$. cincticeps (Wang et al., 2019). RYSV was crudely purified from infected rice plants, as described (Jia et al., 2012). Rabbit polyclonal antibodies against N, P, and M proteins of RYSV, and against $N$. cincticeps ODC1 and OAZ1 were prepared as previously described (Wang et al., 2018). Polyclonal antibodies were conjugated directly to fluorescein isothiocyanate (FITC) or rhodamine according to the manufacturer's instructions.

\section{Yeast two-hybrid assay}

The Matchmaker Gal4 Two-Hybrid System 3 (Clontech, Japan) was used to test the interactions among ODC1, OAZ1, and RYSV M in a yeast two-hybrid test, according to 
the manufacturer's instructions. The M gene of RYSV was amplified and cloned into pGBKT7 vector and pGADT7 vector, respectively, named BD-M and AD-M. Full-length ODC1 was integrated into pGBKT7 vector, named BDODC1. The OAZ1 gene was inserted into pGADT7 vector, named AD-OAZ1. Two OAZ1 deletion fragments, OAZ1-4 (1-188 aa) and OAZ1-5 (84-213 aa) were clones into pGADT7 vector, named AD-OAZ1-4 and AD-OAZ1-5. The recombinant vectors BD-M/AD-OAZ1, BD-M/AD-ODC1, BD-ODC1/AD-OAZ1, BD-ODC1/AD-OAZ1-4; BDODC1/AD-OAZ1-5 and BD-M/AD-OAZ1-4; BD-M/ADOAZ1-5, as well as the positive control pGBKT7-53/ pGADT7-T and negative control pGBKT7-Lam/pGADT7-T, were each co-transformed into the AH109 yeast strain. $\beta$ Galactosidase activity (indicative of successful cloning) was assessed on $\mathrm{SD} /$-Leu/-Trp/-His/-Ade/X-a-gal agar culture medium plates.

\section{GST pull-down assay}

A GST pull-down system was used to validate the interaction between RYSV M and the OAZ1. Briefly, the M gene was amplified and cloned into pGEX-3 encoding a GST tag. The full-length OAZ1 gene was cloned into pET30a for His tag fusion. All constructs were transformed into Escherichia coli strain BL21 and induced as described previously (Wang et al., 2019). The recombinant proteins GST-M and OAZ1-His were purified separately and used in the following pull-down experiment. GST-M was incubated with glutathione-sepharose beads (Amersham, USA) for $4 \mathrm{~h}$ at $4^{\circ} \mathrm{C}$. Beads were rinsed with $0.01 \mathrm{~mol} \mathrm{~L}^{-1}$ PBS to remove unbound proteins and then incubated with purified OAZ1-His recombinant proteins for a further $4 \mathrm{~h}$ at $4^{\circ} \mathrm{C}$. The mixtures were then washed with elution buffer $\left(300 \mathrm{mmol} \mathrm{L}^{-1} \mathrm{NaCl}\right.$, $10 \mathrm{mmol} \mathrm{L}^{-1} \mathrm{Na}_{2} \mathrm{HPO}_{3}, 2.7 \mathrm{mmol} \mathrm{L}^{-1} \mathrm{KCl}$, and $1.7 \mathrm{~mol} \mathrm{~L}^{-1}$ $\mathrm{KH}_{2} \mathrm{PO}_{4}$ ) and subjected to immunoblotting using GST-tag and His-tag antibodies (Abcam, UK), respectively.

\section{UPLC-3Q-MS determination of polyamines}

Viruliferous or non-viruliferous $N$. cincticeps adults (about $2 \mathrm{mg}$ ) were homogenized in ice-cold $\mathrm{HClO}_{4}\left(0.8 \mathrm{~mol} \mathrm{~L}^{-1}\right)$ at a ratio of $20 \mu \mathrm{L} \mathrm{mg}^{-1}$ (volume/mass ratio) using a G50 motor-driven tissue grinder with a plastic grinder head (Sangon Biotech, Shanghai, China) for $1 \mathrm{~min}$, followed by centrifuging at $15,000 \times g$ for $5 \mathrm{~min}$ to extract polyamines. The extract solution was collected and diluted 60-fold with deionized water. The polyamines (including Put, Spd, and $\mathrm{Spm})$ in the final solution were separated and detected by UPLC-3Q-MS. The UPLC-3Q-MS system used in this study consisted of an Agilent 1290 UPLC system and an Agilent 6460 triple quadrupole mass spectrometer (Agilent Technologies, USA). An Agilent ZORBAX HILIC Plus column
$(50 \times 2.1 \mathrm{~mm}, 1.8 \mu \mathrm{m}$ particle size $)$ was employed to achieve separation of the polyamines at a flow rate of $0.5 \mathrm{~mL} \mathrm{~min}^{-1}$. The optimized MS/MS conditions for determination of polyamines were as follows: drying gas temperature $350^{\circ} \mathrm{C}$, gas flow $10 \mathrm{~L} \mathrm{~min}^{-1}$, nebulizer $50 \mathrm{psi}$, capillary voltage $4,000 \mathrm{~V}$ in positive ionization mode, cell accelerator $4 \mathrm{~V}$, and dwell time $50 \mathrm{~ms}$. The entire experiment was repeated three times.

Transcriptional profiling and protein accumulation analysis of ODC1 and OAZ1 in $N$. cincticeps during RYSV infection

To examine ODC1 and OAZ1 gene expression, viruliferous or non-viruliferous $N$. cincticeps adults (20 in each group) were collected and then ground in liquid nitrogen. Total RNAs were extracted and then subjected to RT-qPCR assay using a SYBR Green PCR MasterMix kit (Promega, USA) in a Mastercycler Reaplex4 real-time PCR system (Eppendorf, Germany). For relative quantitation, the transcript levels were normalized using the internal control gene actin and estimated using the $2^{-\Delta \Delta C t}$ (cycle threshold) method. Total proteins were extracted as described previously and analyzed by Western blot assay with rabbit polyclonal antibodies against $\mathrm{N}, \mathrm{M}, \mathrm{ODC} 1$, or OAZ1.

Effects of knockdown of OAZ1 or ODC1 expression by RNAi on the polyamine biosynthesis and viral infection in $R$. dorsalis

The double-strand RNAs of OAZ1 (dsOAZ1, $180 \mathrm{nt}$ ), ODC1 (dsODC1, $230 \mathrm{nt}$ ) and GFP (dsGFP, $210 \mathrm{nt}$ ) genes were synthesized according to the manufacturer's instructions as previously described (Jia et al., 2012). To examine the distribution of RYSV within $N$. cincticeps after knocking down either OAZ1 or ODC1, three groups of second-instar nymphs were microinjected with a mixture of dsRNAs (dsOAZ1, dsODC1 or dsGFP) and RYSV solution, respectively, as described previously (Wang et al., 2019). Six days post-infection, 20 insects from each group were dissected. The internal organs were fixed in $4 \%$ paraformaldehyde in $0.01 \mathrm{~mol} \mathrm{~L}^{-1}$ PBS for at least $8 \mathrm{~h}$ at room temperature and then permeabilized in $4 \%$ Triton X-100 in $0.01 \mathrm{~mol} \mathrm{~L}^{-1}$ PBS buffer for $24 \mathrm{~h}$. The samples were immunolabeled with RYSV N-specific IgG conjugated to rhodamine (N-rhodamine). All samples were then examined using a Leica TCS SP5II confocal microscope.

To examine the polyamine concentrations of RYSV-infected $N$. cincticeps after knocking down either OAZ1 or ODC1, three groups of second-instar nymphs were microinjected with a mixture of dsRNAs (dsOAZ1, dsODC1 or dsGFP) and RYSV solution, respectively. Six days post-infection, $2 \mathrm{mg} N$. cincticeps adults from each group was 
collected and subjected to UPLC-3Q-MS assay following the above conditions. Meanwhile, the total RNAs or proteins were extracted to test the effects of the in vitro synthesized dsRNAs on the transcript or protein levels of N, M, ODA1, or ODC1 by RT-qPCR or Western blot assay, as described above.

\section{In vitro feeding of $N$. cincticeps with polyamines or DFMO}

Five groups of second-instar nymphs (50 in each group) of $N$. cincticeps were first microinjected with RYSV inoculation and then fed in an artificial feeding system using a plastic tube $\left(10 \mathrm{~cm}\right.$ high $\times 5 \mathrm{~cm}$ in diameter) containing $0.1 \mathrm{~g} \mathrm{~mL}^{-1}$ sucrose solution held within a double layer of Parafilm. Feeding assays were performed for 6 days on $10 \%$ sucrose solutions containing either one of three different polyamines with a final concentration of $0.1 \mathrm{mmol} \mathrm{L}^{-1}$ or DFMO with a final concentration of $10 \mathrm{mmol} \mathrm{L}^{-1}$. The survival rate of nymphs was recorded daily. After 4 or 6 days of in vitro ingestion, insects were collected and the effects of DFMO treatment on the production of polyamines Put, Spd and Spm were analyzed by UPLC-3Q-MS system, as described above. Meanwhile, the total RNAs or proteins were extracted to test the effects of the supplement of polyamines or DFMO on the transcript or protein levels of RYSV N, P or M by RT-qPCR or Western blot assay, as described above.

\section{Baculovirus expression of OAZ1, ODC1, and RYSV M in Sf9 cells}

The subcellular localizations of RYSV M, OAZ1, and ODC1 were studied in Spodoptera frugiperda (Sf9) cells using a recombinant baculovirus expression system, as described previously (Wang et al., 2019). Briefly, recombinant baculovirus shuttle vectors expressing M- $6 \times$ His, ODC1-Strep, and OAZ1-Strep were constructed and transformed into $E$. coli DH10 Bac cells (Invitrogen, USA), respectively. Recombinant bacmid DNA containing the expected constructs was separately transfected into Sf9 cells using Cellfectin II (Thermo Fisher Scientific, USA) according to the manufacturer's instructions. Recombinant baculovirus-infected Sf9 cells were incubated for $48 \mathrm{~h}$, then fixed in $4 \%$ paraformaldehyde and immunolabeled with $\mathrm{M}$-specific IgG conjugated to FITC (M-FITC) or rhodamine (M-rhodamine), OAZ1-specific IgG conjugated to FITC (OAZ1-FITC) or rhodamine (OAZ1-rhodamine), or ODC1-speciifc IgG conjugated to rhodamine (ODC1-rhodamine), and then examined using a Leica TCS SP5II confocal microscope.

\section{Statistical analyses}

All data were analyzed using SPSS (version 17.0; SPSS,
USA). For all analyses, a one-tailed Student's $t$ test was used to compare groups, with a significance level of 0.05 . Data were back-transformed after analysis for presentation in the text, figures, and tables.

Compliance and ethics The author(s) declare that they have no conflict of interest.

Acknowledgements We thank members of the Wei lab for stimulating discussions and technical assistance. This research was supported by the National Natural Science Foundation of China (31871931), the Fujian Agriculture and Forestry University Outstanding Young Scientists Project (xjq201705) and National Key Research and Development Project of China (2018YFD0200306).

\section{References}

Beenukumar, R.R., Gödderz, D., Palanimurugan, R., and Dohmen, R.J. (2015). Polyamines directly promote antizyme-mediated degradation of ornithine decarboxylase by the proteasome. Microb Cell 2, 197-207.

Chiu, R.J., Lo, T.C., Pi, C.L., and Chen, M.H. (1965). Transitory yellowing of rice and its transmission by the leafhopper Nephotettix apicalis. Bot Bull Acad Sin Taipei 6, 1-18.

Di, D.P., Zhang, Y.L., Yan, C., Yan, T., Zhang, A.H., Yang, F., Cao, X.L., Li, D.W., Lu, Y.G., Wang, X.B., et al. (2014). First report of Barley yellow striate mosaic virus on wheat in China. Plant Dis 98, 1450.

Fan, H., Zeng, D.S., Ye, B.J., and Lu, B.C. (1965). The preliminary investigation on rice yellow stunt disease in Guangdong. Plant Prot 3, 143-145.

Fang, R.X., Wang, Q., Xu, B.Y., Pang, Z., Zhu, H.T., Mang, K.Q., Gao, D. M., Qin, W.S., and Chua, N.H. (1994). Structure of the nucleocapsid protein gene of rice yellow stunt rhabdovirus. Virology 204, 367-375.

Fang, X.D., Yan, T., Gao, Q., Cao, Q., Gao, D.M., Xu, W.Y., Zhang, Z.J., Ding, Z.H., and Wang, X.B. (2019). A cytorhabdovirus phosphoprotein forms mobile inclusions trafficked on the actin/ER network for viral RNA synthesis. J Exp Bot 70, 4049-4062.

Fauci, A.S., and Morens, D.M. (2016). Zika virus in the Americas-yet another arbovirus threat. N Engl J Med 374, 601-604.

Firpo, M.R., and Mounce, B.C. (2020). Diverse functions of polyamines in virus infection. Biomolecules 10, 628-635.

Fong, W.F., Heller, J.S., and Canellakis, E.S. (1976). The appearance of an ornithine decarboxylase inhibitory protein upon the addition of putrescine to cell cultures. Biochim Biophys Acta Gen Subj 428, 456-465.

Fozard, J.R., Grove, J., Part, M.L., and Prakash, N.J. (1979). Inhibition of early embryogenic development in mice by alpha-difluoromethyl ornithine, an enzyme-activated irreversible inhibitor of L-ornithine decarboxylase. Br J Pharmacol 66, 436P.

Gibson, W., van Breemen, R., Fields, A., LaFemina, R., and Irmiere, A. (1984). D,L-alpha-difluoromethylornithine inhibits human cytomegalovirus replication. J Virol 50, 145-154.

Harty, R.N., Paragas, J., Sudol, M., and Palese, P. (1999). A proline-rich motif within the matrix protein of vesicular stomatitis virus and rabies virus interacts with WW domains of cellular proteins: implications for viral budding. J Virol 73, 2921-2929.

Hibino, H. (1996). Biology and epidemiology of rice viruses. Annu Rev Phytopathol 34, 249-274.

Hogenhout, S.A., Ammar, E.D., Whitfield, A.E., and Redinbaugh, M.G. (2008). Insect vector interactions with persistently transmitted viruses. Annu Rev Phytopathol 46, 327-359.

Huang, Y., Zhao, H., Luo, Z., Chen, X., and Fang, R.X. (2003). Novel structure of the genome of Rice yellow stunt virus: identification of the gene 6-encoded virion protein. J Gen Virol 84, 2259-2264.

Huang, Y.W., Geng, Y.F., Ying, X.B., Chen, X.Y., and Fang, R.X. (2005). Identification of a movement protein of rice yellow stunt rhabdovirus. $\mathrm{J}$ 
Virol 79, 2108-2114

Igarashi, K., and Kashiwagi, K. (2010). Modulation of cellular function by polyamines. Int J Biochem Cell Biol 42, 39-51.

Igarashi, K., and Kashiwagi, K. (2019). The functional role of polyamines in eukaryotic cells. Int J Biochem Cell Biol 107, 104-115.

Jackson, A.O., and Li, Z. (2016). Developments in plant negative-strand RNA virus reverse genetics. Annu Rev Phytopathol 54, 469-498.

Jackson, A.O., Dietzgen, R.G., Goodin, M.M., and Li, Z.H. (2018). Development of model systems for plant rhabdovirus research. Adv Virus Res 102, 23-57.

Jia, D., Guo, N., Chen, H., Akita, F., Xie, L., Omura, T., and Wei, T. (2012). Assembly of the viroplasm by viral non-structural protein Pns10 is essential for persistent infection of rice ragged stunt virus in its insect vector. J Gen Virol 93, 2299-2309.

Kakooza-Mwesige, A., Tshala-Katumbay, D., and Juliano, S.L. (2019). Viral infections of the central nervous system in Africa. Brain Res Bull $145,2-17$.

Kusano, T., Berberich, T., Tateda, C., and Takahashi, Y. (2008). Polyamines: essential factors for growth and survival. Planta 228, 367-381.

Liu, Y., Du, Z., Wang, H., Zhang, S., Cao, M., and Wang, X. (2018). Identification and characterization of wheat yellow striate virus, a novel leafhopper-transmitted nucleorhabdovirus infecting wheat. Front Microbiol 9, 468-478.

Luo, Z., Chen, X., Gao, D., and Fang, R. (1998). The gene 4 of rice yellow stunt rhabdovirus encodes the matrix protein. Virus Genes 16, 277-280.

Luo, Z.L., and Fang, R.X. (1998). Structure analysis of the rice yellow stunt rhabdovirus glycoprotein gene and its mRNA. Arch Virol 143, 24532459.

Matsufuji, S., Matsufuji, T., Miyazaki, Y., Murakami, Y., Atkins, J.F., Gesteland, R.F., and Hayashi, S. (1995). Autoregulatory frameshifting in decoding mammalian ornithine decarboxylase antizyme. Cell 80, 5160.

Metcalf, B., Bey, P., Danzin, C., Jung, M., Casara, P.L., and Vevert, J. (1978). Catalytic irreversible inhibition of mammalian ornithine decarboxylase by substrate and product analogs. J Am Chem Soc 100, 2251-2252.

Michael, A.J. (2016). Polyamines in eukaryotes, bacteria, and archaea. J Biol Chem 291, 14896-14903.

Morehead, T.A., Gurnon, J.R., Adams, B., Nickerson, K.W., Fitzgerald, L. A., and Van Etten, J.L. (2002). Ornithine decarboxylase encoded by chlorella virus PBCV-1. Virology 301, 165-175.

Morrison, T.E. (2014). Reemergence of Chikungunya virus. J Virol 88, $11644-11647$.

Mounce, B.C., Poirier, E.Z., Passoni, G., Simon-Loriere, E., Cesaro, T., Prot, M., Stapleford, K.A., Moratorio, G., Sakuntabhai, A., Levraud, J. P., et al. (2016a). Interferon-induced spermidine-spermine acetyltransferase and polyamine depletion restrict Zika and Chikungunya viruses. Cell Host Microbe 20, 167-177.

Mounce, B.C., Cesaro, T., Moratorio, G., Hooikaas, P.J., Yakovleva, A., Werneke, S.W., Smith, E.C., Poirier, E.Z., Simon-Loriere, E., Prot, M., et al. (2016b). Inhibition of polyamine biosynthesis is a broad-spectrum strategy against RNA viruses. J Virol 90, 9683-9692.
Mounce, B.C., Olsen, M.E., Vignuzzi, M., and Connor, J.H. (2017). Polyamines and their role in virus infection. Microbiol Mol Biol Rev 81, e00029.

Okumura, A., and Harty, R.N. (2011). Rabies virus assembly and budding. Adv Virus Res 79, 23-32.

Olsen, M.E., Filone, C.M., Rozelle, D., Mire, C.E., Agans, K.N., Hensley, L., and Connor, J.H. (2016). Polyamines and hypusination are required for Ebolavirus gene expression and replication. mBio 7, e00882-16.

Palanimurugan, R., Scheel, H., Hofmann, K., and Jürgen Dohmen, R. (2014). Polyamines regulate their synthesis by inducing expression and blocking degradation of ODC antizyme. EMBO J 23, 4857-4867.

Pegg, A.E. (2008). Spermidine/spermine- $N^{1}$-acetyltransferase: a key metabolic regulator. Am J Physiol-Endocrinol Metab 294, E995-E1010.

Pohjanpelto, P., Sekki, A., Hukkanen, V., and von Bonsdorff, C.H. (1988). Polyamine depletion of cells reduces the infectivity of herpes simplex virus but not the infectivity of Sindbis virus. Life Sci 42, 2011-2018.

Finke, S., and Conzelmann, K.K. (2003). Dissociation of rabies virus matrix protein functions in regulation of viral RNA synthesis and virus assembly. J Virol 77, 12074-12082.

Tabor, C.W., and Tabor, H. (1984). Polyamines. Annu Rev Biochem 53, 749-790.

Tuomi, K., Raina, A., and Mäntyjärvi, R. (1982). Synthesis of Semlikiforest virus in polyamine-depleted baby-hamster kidney cells. Biochem J 206, 113-119.

Tyms, A.S., Scamans, E., and Williamson, J.D. (1979). Polyamine metabolism in MRC5 cells infected with different herpesviruses. Biochem Biophys Res Commun 86, 312-318.

Wang, H., Wang, J., Xie, Y., Fu, Z., Wei, T., and Zhang, X.F. (2018). Development of leafhopper cell culture to trace the early infection process of a nucleorhabdovirus, rice yellow stunt virus, in insect vector cells. Virol J 15, 72-82.

Wang, H., Wang, J., Zhang, Q., Zeng, T., Zheng, Y., Chen, H., Zhang, X.F., and Wei, T. (2019). Rice yellow stunt nucleorhabdovirus matrix protein mediates viral axonal transport in the central nervous system of its insect vector. Front Microbiol 10, 939-951.

Wang, H., Liu, Y., Mo, L., Huo, C., Wang, Z., Zhong, P., Jia, D., Zhang, X., Chen, Q., Chen, H., et al. (2020). A neuron-specific antiviral mechanism modulates the persistent infection of rice rhabdoviruses in leafhopper vectors. Front Microbiol 11, 513-523.

Wei, T., and Li, Y. (2016). Rice reoviruses in insect vectors. Annu Rev Phytopathol 54, 99-120.

Williamson, J.D. (1976). The effect of methylglyoxal bis(guanylhydrazone) on vaccinia virus replication. Biochem Biophys Res Commun 73, 120126.

Yang, X., Huang, J., Liu, C., Chen, B., Zhang, T., and Zhou, G. (2017). Rice stripe mosaic virus, a novel cytorhabdovirus infecting rice via leafhopper transmission. Front Microbiol 7, 2140-2152.

Yang, X., Chen, B., Zhang, T., Li, Z., Xu, C., and Zhou, G. (2018). Geographic distribution and genetic diversity of rice stripe mosaic virus in southern China. Front Microbiol 9, 3068-3081.

Zhou, X., Lin, W., Sun, K., Wang, S., Zhou, X., Jackson, A.O., and Li, Z. (2019). Specificity of plant rhabdovirus cell-to-cell movement. J Virol 93, e00296-19.

\section{SUPPORTING INFORMATION}

The supporting information is available online at https://doi.org/10.1007/s11427-020-1846-8. The supporting materials are published as submitted, without typesetting or editing. The responsibility for scientific accuracy and content remains entirely with the authors. 